\title{
Prevalencia y características de objetores de conciencia a la Ley 21.030 en instituciones públicas
}

\section{Prevalence and characteristics of conscientious objectors to Law 21.030 in public institutions}

\author{
Adela Montero ${ }^{1 *}$, Mirliana Ramírez-Pereira², Paz Robledo ${ }^{3}$, Lidia Casas ${ }^{4}$, Lieta Vivaldi5, \\ Temístocles Molina y Daniela González ${ }^{\uparrow}$
}

${ }^{1}$ Centro de Medicina Reproductiva y Desarrollo Integral del Adolescente, Facultad de Medicina, Universidad de Chile; ${ }^{2}$ Departamento de Enfermería, Facultad de Medicina, Universidad de Chile; ${ }^{3}$ Servicio de Pediatría y Cirugía Infantil, Unidad de Cuidados Medios, Hospital La Florida Dra. Eloísa Díaz; ${ }^{4}$ Centro de Derechos Humanos, Facultad de Derecho, Universidad Diego Portales; ${ }^{5}$ Departamento de Ciencias del Derecho, Facultad de Derecho, Universidad Alberto Hurtado. Santiago, Chile

\section{Resumen}

Introducción: La Ley 21.030 permite la objeción de conciencia al personal de salud al interior del pabellón y a las instituciones privadas. Ha sido considerada conflicto de intereses no monetario, al anteponer los valores personales, afectando el cumplimiento del deber profesional. Objetivos: Establecer la prevalencia de funcionarios/as objetores/as en los hospitales de la red pública del país y caracterizarles según edad, género y nacionalidad. Método: Estudio cuantitativo, analítico y transversal. Se utilizaron medidas de tendencia central y dispersión. Para medir la asociación entre variables sociodemográficas, profesión y causal objetada, se utilizaron las pruebas de $\chi^{2}$, exacta de Fisher y de Kruskal-Wallis. Resultados: En 57 hospitales, se observa una mayor frecuencia de objetores en causal 3. En 443 objetores, la mediana de edad fue de 43 años, el 64,8\% mujeres y el $87,4 \%$ de nacionalidad chilena. En las zonas centro y sur del país se concentra la mayor proporción de hospitales con más del 50\% de objetores. Conclusiones: La dificultad para obtener información impide conocer cabalmente la magnitud de la objeción de conciencia. Resulta preocupante la alta prevalencia de objetores, específicamente en la causal violación. La objeción no puede operar como barrera que vulnere los derechos y la dignidad de las mujeres.

Palabras clave: Objeción de conciencia. Aborto. Derechos sexuales y reproductivos. Ley 21.030. Compromiso de conciencia.

\section{Abstract}

Introduction: Law 21.030 incorporates conscientious objection for health personnel inside the surgical ward and allows its invocation by private institutions. It has been considered a conflict of interest, not monetary, by putting personal values first, affecting the fulfillment of professional duty. Objective: To establish the prevalence of objectors in the country's public network hospitals and characterize them according to age, gender, and nationality. Method: Quantitative, analytical, and cross-sectional study. Central and dispersion trend measures were used. For measuring the association between sociodemographic variables, profession and causal objected, test $\chi^{2}$, Fisher exact and Kruskal-Wallis test were used. Results: In 57 hospitals, a higher frequency of objectors were observed in the third causal. In 443 objectors, the median age was 43 years, $64.8 \%$ are women, and $87.4 \%$ are Chilean. The central and southern areas of the country have the highest proportion of hospitals, with more than 50\% objectors. Conclusions: The difficulty for obtaining the information prevents fully knowing the magnitude of conscientious objection in Chile. The high prevalence of objectors, specifically in the causal violation is worrying. The conscientious objection cannot operate as a barrier that violates the rights and dignity of women.

Keywords: Conscientious objection. Abortion. Sexual and reproductive rights. Law 21.030. Conscientious commitment.

Correspondencia:

*Adela Montero

E-mail: amontero@uchile.cl
Fecha de recepción: 26-07-2021

Fecha de aceptación: 29-11-2021

DOI: 10.24875/RECHOG.21000006
Disponible en internet: 07-02-2022 Rev Chil Obstet Ginecol. 2021;86(6):521-528 www. rechog.com

0048-766X / ๑ 2021 Sociedad Chilena de Obstetricia y Ginecología. Publicado por Permanyer. Este es un artículo open access bajo la licencia CC BY-NC-ND (https://creativecommons.org/licenses/by-nc-nd/4.0/). 


\section{Introducción}

Después de casi tres décadas de la derogación del aborto terapéutico, el 14 de septiembre de 2017 se promulgó en Chile la Ley 21.030, que despenalizó la interrupción voluntaria del embarazo (IVE), en tres causales:

- Causal 1: la mujer se encuentre en riesgo vital, de modo que la interrupción del embarazo evite un peligro para su vida.

- Causal 2: el embrión o feto padezca patología congénita adquirida o genética letal, incompatible con la vida extrauterina independiente.

- Causal 3: embarazo resultado de violación'.

Durante la tramitación legislativa de la ley, el intento de posicionar la objeción de conciencia como un derecho para el/la profesional requerido/a para realizar la interrupción, su ampliación para profesionales que indirectamente participaran del procedimiento y su invocación por las instituciones, fue el resultado de un amplio y complejo debate ${ }^{2}$, que fue zanjado con la sentencia del Tribunal Constitucional, organismo extraparlamentario, que extendió la objeción de conciencia a profesionales y técnicos con desempeño en pabellones quirúrgicos y a las instituciones de salud ${ }^{3}$. Posterior a su promulgación, se derogaron dos protocolos que regulaban su ejercicio ${ }^{4-6}$. Finalmente, siguiendo el dictamen de la Contraloría General de la República, el 23 de octubre de 2018 se publicó el Reglamento, reflejando la complejidad de la objeción de conciencia en Chile?.

En salud, resulta complejo encontrar una definición asertiva, debido a las diferentes posturas ideológicas, a su consideración o no como derecho y a su vinculación o diferenciación con la desobediencia civil. Podemos definirla como la exención del cumplimiento de una obligación jurídica, derivada de las relaciones laborales o funcionarias, individuales, frente a una obligación personal, esgrimiendo razones religiosas o filosóficas $^{8}$. Giubilini y Savulescu ${ }^{9}$ la consideran un conflicto de intereses no monetario, derivado de la anteposición personal de valores morales o religiosos de la profesional de la salud, afectando negativamente su obligación de proveer el cuidado y la atención a la población que lo requiere.

Referente a su ejercicio, su inadecuada invocación, especialmente en Latinoamérica, se ha traducido en una barrera significativa para acceder al aborto legal y seguro $^{10,11}$. Un ejemplo ocurrió en Salto (Uruguay), donde posterior a la promulgación de la ley el 100\% de los gineco-obstetras se declararon objetores, debiendo recurrir al traslado de profesionales desde otras localidades para asegurar las prestaciones ${ }^{11}$.

En Chile, aun cuando el Reglamento no señala que deba fundamentarse la objeción, establece límites precisos para el ejercicio individual e institucional. Como no es posible incluir de manera completa los aspectos vinculados con la regulación, se recomienda revisar este Reglamento.

Para la objeción individual, al cierre de este manuscrito (18/08/2021) solo se dispone de datos oficiales a septiembre de 2019, correspondientes a los 29 servicios de salud públicos ${ }^{12}$. En junio de 2018, de 1140 gineco-obstetras contratados en estos servicios de salud, el $20,3 \%$ objetaban en caso de riesgo vital de la mujer (causal 1), el 27,3\% frente a patología embrionaria/fetal congénita 0 genética letal (causal 2), y el $47,2 \%$ en embarazo por violación (causal 3) ${ }^{13}$. En 2019, el porcentaje de objetores tiende a disminuir levemente: de 1214 gineco-obstetras contratados, el $18,4 \%$ objeta en la causal 1 , el $25,3 \%$ en la causal 2 y el $46 \%$ en la causal $3^{12}$ (Tabla 1). Considerando una proporción de gineco-obstetras objetores superior al $50 \%$, en las causales 1 y 2 hay dos servicios de salud, respectivamente, mientras que en la causal 3 hay 12 servicios de salud. Los servicios de salud Maule y Araucanía Norte presentan cifras mayores del $50 \%$ para todas las causales ${ }^{12}$.

En otros actores, en junio de 2018 , el $9,3 \%$ de profesionales anestesistas objetan en la causal 1, el $13,7 \%$ en la causal 2 y el $21,7 \%$ en la causal 3 . El $12 \%$ de profesionales no médicos objetan en la causal 1 , el $18,4 \%$ en la causal 2 y el $20,7 \%$ en la causal 3 . Del personal técnico de enfermería de nivel superior (TENS), el 10,6\% objetan en la causal 1 , el $11,9 \%$ en la causal 2 y el 13,2\% en la causal $3^{13}$. En 2019, entre los/las anestesistas se observa una leve tendencia al aumento para la causal 3 y se mantienen los porcentajes para las causales 1 y 2 . En profesionales no médicos se observa prácticamente la misma proporción de objetores en la causal 3 y disminuye en las otras causales. Los/las TENS disminuyen la cantidad de objetores en todas las causales ${ }^{12}$ (Tabla 1).

Desde la entrada en vigencia de la ley, cinco instituciones privadas confesionales objetan en todas las causales y una institución no confesional objeta en la causal $3^{14}$.

Aparte de esta información, no hemos identificado estudios ni publicaciones que caractericen a funcionarios/as objetores/as de conciencia a la IVE en Chile. Por ello, el objetivo de este estudio es identificar la prevalencia de objetores/as de conciencia que se desempeñan en los hospitales de la red pública del país y 
Tabla 1. Prevalencia de objetores/as de conciencia por servicios de salud. Chile, junio de 2018 y septiembre de 2019

\begin{tabular}{|c|c|c|c|c|c|c|c|c|}
\hline \multirow[t]{3}{*}{ Profesión } & \multicolumn{4}{|c|}{ Junio 2018} & \multicolumn{4}{|c|}{ Septiembre 2019} \\
\hline & \multirow[t]{2}{*}{ Contratados/as (n) } & \multicolumn{3}{|c|}{ Causales (\%) } & \multirow[t]{2}{*}{ Contratados/as (n) } & \multicolumn{3}{|c|}{ Causales (\%) } \\
\hline & & 1 & 2 & 3 & & 1 & 2 & 3 \\
\hline Gineco-obstetras & 1140 & 20,3 & 27,3 & 47,2 & 1214 & 18,4 & 25,3 & 46 \\
\hline Anestesistas & 729 & 9,3 & 13,7 & 21,7 & 823 & 9 & 13,5 & 22,5 \\
\hline Profesionales no médicos & 1019 & 12 & 18,4 & 20,7 & 1018 & 9,4 & 15,7 & 20,8 \\
\hline TENS & 1768 & 10,6 & 11,9 & 13,2 & 1754 & 8 & 9,6 & 11,9 \\
\hline
\end{tabular}

TENS: técnicas/os de enfermería de nivel superior.

Fuente: elaboración propia a partir de Funcionarios objetores de conciencia por Servicios de Salud. Ministerio de Salud de Chile (https://www.minsal.cl/funcionariosobjetores-de-conciencia-por-servicio-de-salud/).

caracterizarles según la información disponible en el formulario de manifestación de objeción de conciencia.

\section{Método}

Estudio cuantitativo, analítico y transversal. De mayo a octubre de 2020 se solicitó por Portal de Transparencia información a las siguientes instituciones reguladas por la Ley $20.285^{15}$ : Subsecretaría de Salud Pública y de Redes Asistenciales, Servicios de Salud, Hospitales Públicos y Secretarías Regionales Ministeriales de Salud. Se elaboró una pauta para recabar información desde septiembre de 2017 hasta junio de 2020: número y porcentaje de objetores/as respecto del total de funcionarios/ as contratados/as, profesión, causal(es) objetada(s), género, edad, nacionalidad, extensión a causal no prevista y retractación de la objeción.

Se realizó un análisis descriptivo con medidas de tendencia central y dispersión para caracterizar la muestra. Se midió la asociación entre las variables sociodemográficas, la profesión y la(s) causal(es) objetada(s). Se utilizaron las pruebas de $\chi^{2}$, exacta de Fisher y de Kruskal-Wallis, y el software estadístico STATA v 12 (StataCorp LP, Texas, USA). Se consideró significativo un valor de $p<0,05$.

Los resultados derivan del Proyecto Fondecyt Regular n. ${ }^{\circ}$ 1200374, aprobado por el Comité de Ética de Investigación en Seres Humanos, Facultad de Medicina, Universidad de Chile (Acta $n . \stackrel{009}{0}$ ).

\section{Resultados}

Los datos corresponden a información recibida al $31 / 03 / 2021$ sobre objetores/as a la IVE que cumplen funciones en hospitales adscritos a la red pública. La entrega de la información por los organismos regulados ameritó habitualmente prórrogas o derivación de la solicitud hacia otras instituciones, dando cuenta que los datos no se encontraban centralizados o sistematizados. No fue posible obtener información de todos los establecimientos de salud públicos del país. Varias instituciones denegaron su entrega aduciendo diversas causas: falta de personal para recopilar la información (especialmente durante la pandemia es por el virus denominado SARSCoV-2), causal de secreto o reserva, o gran cantidad de actos administrativos para responder al requerimiento. Considerando la disparidad de la información recibida por año de ocurrencia, se exponen resultados actualizados a junio de 2020.

\section{Prevalencia de la objeción de conciencia}

La tabla 2 muestra la distribución de objetores/as según profesión y causal en que objetan, respecto del total de funcionarios/as contratados/as. Los datos corresponden a 57 hospitales públicos que realizan prestaciones vinculadas con la Ley 21.030. Se excluyó una institución por información no consistente. Cabe señalar que una persona puede objetar en una, en más de una o en todas las causales. De 4378 funcionarios/ as contratados/as, el $12,7 \%$ objeta en la causal 1 (riesgo vital de la gestante), el $17 \%$ en la causal 2 (patología congénita o genética embrionaria/fetal letal) y el $25,7 \%$ en la causal 3 (embarazo por violación). Del total de funcionarios/as, la causal 3 tiene mayor porcentaje de objeción, con más frecuencia en gineco-obstetras (49\%), mientras que para la causal 1 hay un menor porcentaje de objetores. 
Tabla 2. Distribución de objetores/as según profesión y causal en la que objetan respecto del total de funcionarios/ as contratados/as en 57 hospitales públicos. Chile, junio de 2020

\begin{tabular}{|c|c|c|c|c|c|c|c|c|c|c|}
\hline Profesión & Gine & 0-obstetras & & tesistas & Prot & $\begin{array}{l}\text { onales no } \\
\text { dicos }\end{array}$ & & TENS & & Total \\
\hline \multirow[t]{2}{*}{ Total funcionarios/as (n) } & \multicolumn{2}{|r|}{1103} & \multicolumn{2}{|c|}{721} & \multicolumn{2}{|c|}{1041} & \multicolumn{2}{|r|}{1513} & \multicolumn{2}{|c|}{4378} \\
\hline & n & $\begin{array}{c}\% \\
\text { (IC95\%) }\end{array}$ & n & $\begin{array}{c}\% \\
\text { (IC95\%) }\end{array}$ & n & $\begin{array}{c}\% \\
\text { (IC95\%) }\end{array}$ & $\mathrm{n}$ & $\begin{array}{c}\% \\
\text { (IC95\%) }\end{array}$ & n & $\begin{array}{c}\% \\
\text { (IC95\%) }\end{array}$ \\
\hline Causal 1 & 210 & $\begin{array}{c}19 \\
(16,8-21,5)\end{array}$ & 81 & $\begin{array}{c}11,2 \\
(9,0-13,8)\end{array}$ & 105 & $\begin{array}{c}10,1 \\
(8,3-12,1)\end{array}$ & 162 & $\begin{array}{c}10,7 \\
(9,2-12,4)\end{array}$ & 558 & $\begin{array}{c}12,7 \\
(11,8-13,8)\end{array}$ \\
\hline Causal 2 & 304 & $\begin{array}{c}27,6 \\
(24,9-30,3)\end{array}$ & 101 & $\begin{array}{c}14 \\
(11,6-16,8)\end{array}$ & 139 & $\begin{array}{c}13,4 \\
(11,3-15,6)\end{array}$ & 200 & $\begin{array}{c}13,2 \\
(11,6-15,0)\end{array}$ & 744 & $\begin{array}{c}17 \\
(15,9-18,1)\end{array}$ \\
\hline Causal 3 & 540 & $\begin{array}{c}49 \\
(46,0-52,0)\end{array}$ & 160 & $\begin{array}{c}22,2 \\
(19,2-25,4)\end{array}$ & 188 & $\begin{array}{c}18,1 \\
(15,8-20,5)\end{array}$ & 238 & $\begin{array}{c}15,7 \\
(13,9-17,7)\end{array}$ & 1126 & $\begin{array}{c}25,7 \\
(24,4-27,0)\end{array}$ \\
\hline
\end{tabular}

IC95\%: intervalo de confianza del 95\%; TENS: técnicas/os de enfermería de nivel superior. Elaboración propia a partir de información obtenida por Ley 20.285.

La tabla 3 expone datos específicos de 443 objetores de 21 hospitales públicos, que proporcionaron información según la(s) causal(es) en que objetan. La primera parte comprende datos globales para las distintas causales, pudiendo haber objeción en más de una. Así, quienes objetan en la causal 1 son el $54,2 \%$, y en la causal 2 son el $68,6 \%$. En ambas causales se observa un mayor porcentaje en profesionales no médicos y TENS: $57,5 \%$ y $63,6 \%(p=0,026)$ y $79,5 \%$ y $78 \%(p=0,001)$, respectivamente. La mayoría de las objeciones ocurren en la causal $3(94,8 \%)$, siendo mayor en gineco-obstetras $(97,9 \%)$ y profesionales no médicos $(94,5 \%)(p=0,023)$. Al desglosar por causales en que cada persona manifiesta su objeción, quienes objetan solo en la causal 1 representan un bajo porcentaje del total de la muestra $(1,4 \%)$, observándose una mayor frecuencia en anestesistas $(6,5 \% ; p=$ $0,030)$. Para objetores solo en la causal $2(2,3 \%)$, así como para objetores en las causales 1 y $2(1,6 \%)$, en las causales 1 y $3(1,4 \%)$, y en las causales 2 y $3(14,9 \%)$, no se observa diferencia significativa según la profesión. La objeción solo en la causal 3 representa un $28,7 \%$ de objetores, siendo nuevamente mayor en gineco-obstetras (38\%) y anestesistas $(28,3 \%)(p=$ $0,001)$. En todas las causales, la objeción constituye un $49,9 \%$ del total, con mayor porcentaje en profesionales no médicos $(56,2 \%)$ y TENS $(58,3 \%)(p=0,028)$.

\section{Edad, género y nacionalidad}

La tabla 4 muestra los datos de 21 hospitales públicos. Algunas instituciones solo informaron edad y género, otras informaron género y nacionalidad, y otras solo género. Para un total de 443 objetores, el 43,3\% son gineco-obstetras, el 10,4\% anestesistas, el 16,5\% profesionales no médicos y el $29,8 \%$ TENS o auxiliares de enfermería. En 320 objetores, la mediana de edad es de 43 años (rango: 23-73 años). Las/los gineco-obstetras presentan una mediana de edad mayor que las/ los profesionales no médicos y las/los TENS (49 años frente a 43 y 38 años, respectivamente). En anestesistas se observa una mayor edad que en TENS (46 años frente 38 años) $(p<0,05)$. Respecto del género, en 426 objetores, el $63,8 \%$ son mujeres. Las/los profesionales no médicos y TENS agrupan un mayor número de mujeres: $88,9 \%$ y $95,9 \%$, respectivamente $(p=0,001)$. En 374 objetores, el 87,4\% son de nacionalidad chilena. En profesionales no médicos y TENS, el $100 \%$ son de nacionalidad chilena. En gineco-obstetras y anestesistas se observa una mayor proporción de objetores de nacionalidad venezolana, con un $14,8 \%$ y un $37,5 \%$, respectivamente $(p=0,001)$.

\section{Accesibilidad a la IVE}

Según el porcentaje de objetores, es factible monitorear la accesibilidad a la IVE en los establecimientos de salud. Siguiendo la clasificación de Maturana ${ }^{16}$, agruparemos los establecimientos en cuatro categorías: accesibilidad alta, 0 a $25 \%$ de objetores; accesibilidad media, 25,1 a $50 \%$ de objetores; accesibilidad baja, 50,1 a 75\% de objetores; y accesibilidad restringida o nula, 75,1 a 100\% de objetores. En 57 hospitales, la mayor diferencia se presenta en gineco-obstetras. Con una proporción de objetores mayor del $50 \%$ hay cinco establecimientos en la causal 1 , ocho en la 
Tabla 3. Objetores/as de conciencia según profesión y causal en la que objetan en 21 hospitales públicos. Chile, junio de 2020

\begin{tabular}{|c|c|c|c|c|c|c|}
\hline Profesión & Gineco-obstetras & Anestesistas & $\begin{array}{l}\text { Profesionales no } \\
\text { médicos }\end{array}$ & TENS & Total & $\mathbf{p}$ \\
\hline n & $n=192$ & $n=46$ & $n=73$ & $\mathrm{n}=132$ & $n=443$ & \\
\hline Causal 1 & $\begin{array}{c}90 \\
(46,9 \%)\end{array}$ & $\begin{array}{c}24 \\
(52,2 \%)\end{array}$ & $\begin{array}{c}42 \\
(57,5 \%)\end{array}$ & $\begin{array}{c}84 \\
(63,6 \%)\end{array}$ & $\begin{array}{c}240 \\
(54,2 \%)\end{array}$ & 0,026 \\
\hline Causal 2 & $\begin{array}{c}114 \\
(59,4 \%)\end{array}$ & $\begin{array}{c}29 \\
(63 \%)\end{array}$ & $\begin{array}{c}58 \\
(79,5 \%)\end{array}$ & $\begin{array}{c}103 \\
(78 \%)\end{array}$ & $\begin{array}{c}304 \\
(68,6 \%)\end{array}$ & 0,001 \\
\hline Causal 3 & $\begin{array}{c}188 \\
(97,9 \%)\end{array}$ & $\begin{array}{c}41 \\
(89,1 \%)\end{array}$ & $\begin{array}{c}69 \\
(94,5 \%)\end{array}$ & $\begin{array}{c}122 \\
(92,4 \%)\end{array}$ & $\begin{array}{c}420 \\
(94,8 \%)\end{array}$ & 0,023 \\
\hline Solo causal 1 & $\begin{array}{c}1 \\
(0,5 \%)\end{array}$ & $\begin{array}{c}3 \\
(6,5 \%)\end{array}$ & $\begin{array}{c}0 \\
(0 \%)\end{array}$ & $\begin{array}{c}2 \\
(1,5 \%)\end{array}$ & $\begin{array}{c}6 \\
(1,4 \%)\end{array}$ & 0,030 \\
\hline Solo causal 2 & $\begin{array}{c}1 \\
(0,5 \%)\end{array}$ & $\begin{array}{c}2 \\
(4,3 \%)\end{array}$ & $\begin{array}{c}3 \\
(4,1 \%)\end{array}$ & $\begin{array}{c}4 \\
(3 \%)\end{array}$ & $\begin{array}{c}10 \\
(2,3 \%)\end{array}$ & 0,072 \\
\hline Solo causal 3 & $\begin{array}{c}73 \\
(38 \%)\end{array}$ & $\begin{array}{c}13 \\
(28,3 \%)\end{array}$ & $\begin{array}{c}15 \\
(20,5 \%)\end{array}$ & $\begin{array}{c}26 \\
(19,7 \%)\end{array}$ & $\begin{array}{c}127 \\
(28,7 \%)\end{array}$ & 0,001 \\
\hline Causales 1 y 2 & $\begin{array}{c}2 \\
(1 \%)\end{array}$ & $\begin{array}{c}0 \\
(0 \%)\end{array}$ & $\begin{array}{c}1 \\
(1,4 \%)\end{array}$ & $\begin{array}{c}4 \\
(3 \%)\end{array}$ & $\begin{array}{c}7 \\
(1,6 \%)\end{array}$ & 0,449 \\
\hline Causales 1 y 3 & $\begin{array}{c}4 \\
(2,1 \%)\end{array}$ & $\begin{array}{c}1 \\
(2,2 \%)\end{array}$ & $\begin{array}{c}0 \\
(0 \%)\end{array}$ & $\begin{array}{c}1 \\
(0,8 \%)\end{array}$ & $\begin{array}{c}6 \\
(1,4 \%)\end{array}$ & 0,507 \\
\hline Causales 2 y 3 & $\begin{array}{c}28 \\
(14,6 \%)\end{array}$ & $\begin{array}{c}7 \\
(15,2 \%)\end{array}$ & $\begin{array}{c}13 \\
(17,8 \%)\end{array}$ & $\begin{array}{c}18 \\
(13,6 \%)\end{array}$ & $\begin{array}{c}66 \\
(14,9 \%)\end{array}$ & 0,880 \\
\hline Todas las causales & $\begin{array}{c}83 \\
(43,2 \%)\end{array}$ & $\begin{array}{c}20 \\
(43,5 \%)\end{array}$ & $\begin{array}{c}41 \\
(56,2 \%)\end{array}$ & $\begin{array}{c}77 \\
(58,3 \%)\end{array}$ & $\begin{array}{c}221 \\
(49,9 \%)\end{array}$ & 0,028 \\
\hline
\end{tabular}

TENS: técnicas/os de enfermería de nivel superior.

Fuente: elaboración propia a partir de información obtenida por Ley 20.285.

causal 2 y 31 en la causal 3 , que estarían representando accesibilidad baja o restringida (Tabla 5).

La figura 1 muestra la georreferenciación por regiones. Para el total de funcionarios/as, 35 hospitales públicos tienen más del $50 \%$ de objetores/as, concentrándose en las zonas centro y sur del país.

\section{Extensión a causal no prevista o retractación}

Treinta y seis hospitales no poseen esta información. De quienes respondieron, no hubo extensión a causal no prevista y hubo dos casos de retractación.

\section{Discusión}

Resulta preocupante la alta prevalencia de objetores, específicamente en la causal de embarazo por violación, en funcionarios/as que se desempeñan en hospitales públicos, responsables de la atención de salud de la mayoría de nuestra población. Si consideramos que durante el período 2018-2020 hubo solo 414 casos en la causal $3^{17}$, cifra muy inferior a la estimada, al vincularla con la alta proporción de objetores para esta causal, es posible inferir que la objeción de conciencia podría estar actuando como una barrera en los tres niveles de atención. También preocupa el número global de objetores en la causal 1, al relacionarse directamente con el riesgo vital de la mujer y porque durante la discusión de la ley se argumentaba que no ameritaba legislar, porque las interrupciones se realizaban siguiendo la lex $\operatorname{artis}^{18}$. Un estudio con docentes gineco-obstetras y matronas/es que se manifestaron contrarios al aborto consideró que en esta causal no podría invocarse la objeción de conciencia ${ }^{19}$.

Datos de actores relevantes que participaron en el debate y la tramitación de la Ley 21.030 permiten identificar diversos argumentos para objetar. Globalmente, la objeción se sustentaría en la libertad de conciencia, pensamiento y religión. El fundamento religioso considera el respeto de la vida humana, desde la concepción hasta la muerte natural. También habría temor a 
Tabla 4. Caracterización de objetores/as de conciencia por profesión, edad, género y nacionalidad en 21 hospitales públicos. Chile, junio de 2020

\begin{tabular}{|c|c|c|c|c|c|c|c|}
\hline \multirow{2}{*}{\multicolumn{2}{|c|}{ Variable }} & Profesión & $\begin{array}{l}\text { Gineco- } \\
\text { obstetras } \\
\text { (1) }\end{array}$ & $\begin{array}{c}\text { Anestesistas } \\
\text { (2) }\end{array}$ & $\begin{array}{l}\text { Profesionales } \\
\text { no médicos } \\
\text { (3) }\end{array}$ & $\begin{array}{l}\text { TENS } \\
\text { (4) }\end{array}$ & Total \\
\hline & & $\mathrm{n}$ & $n=192$ & $n=46$ & $n=73$ & $n=132$ & $n=443$ \\
\hline \multirow[t]{4}{*}{ Edad } & Mediana (años) & \multirow{4}{*}{$\begin{array}{l}\text { Gineco-obstetras: } 129 \\
\text { Anestesistas: } 29 \\
\text { Profesionales no } \\
\text { médicos: } 67 \\
\text { TENS: } 95\end{array}$} & 49 & 46 & 43 & 38 & 43 \\
\hline & Min-máx (años) & & $29-73$ & $27-69$ & $24-68$ & $23-63$ & $23-73$ \\
\hline & Rango intercuartílico & & 21 & 16 & 20 & 18 & 20 \\
\hline & $\mathrm{p}$ & & \multicolumn{5}{|c|}{$1-3 ; 1-4 ; 2-4^{*}$} \\
\hline \multirow[t]{3}{*}{ Género } & Femenino & \multirow{3}{*}{$\begin{array}{c}\text { Gineco-obstetras: } 187 \\
\text { Anestesistas: } 44 \\
\text { Profesionales no } \\
\text { médicos: } 72 \\
\text { TENS: } 123\end{array}$} & $\begin{array}{c}71 \\
(38 \%)\end{array}$ & $\begin{array}{c}19 \\
(43,2 \%)\end{array}$ & $64(88,9 \%)$ & $\begin{array}{c}118 \\
(95,9 \%)\end{array}$ & $\begin{array}{c}272 \\
(63,8 \%)\end{array}$ \\
\hline & Masculino & & $\begin{array}{c}116 \\
(62 \%)\end{array}$ & $\begin{array}{c}25 \\
(56,8 \%)\end{array}$ & $\begin{array}{c}8 \\
(11,1 \%)\end{array}$ & $\begin{array}{c}5 \\
(4,1 \%)\end{array}$ & $\begin{array}{c}154 \\
(36,2 \%)\end{array}$ \\
\hline & $\mathrm{p}$ & & & & 0,001 & & \\
\hline \multirow[t]{4}{*}{ Nacionalidad } & Chilena & \multirow{3}{*}{$\begin{array}{c}\text { Gineco-obstetras: } 169 \\
\text { Anestesistas: } 40 \\
\text { Profesionales no } \\
\text { médicos: } 61 \\
\text { TENS: } 104\end{array}$} & $\begin{array}{c}137 \\
(81,1 \%)\end{array}$ & $\begin{array}{c}25 \\
(62,5 \%)\end{array}$ & $61(100 \%)$ & $\begin{array}{c}104 \\
(100 \%)\end{array}$ & $\begin{array}{c}327 \\
(87,4 \%)\end{array}$ \\
\hline & Venezolana & & $\begin{array}{c}25 \\
(14,8 \%)\end{array}$ & $\begin{array}{c}15 \\
(37,5 \%)\end{array}$ & 0 & 0 & $\begin{array}{c}40 \\
(10,7 \%)\end{array}$ \\
\hline & Otra & & $\begin{array}{c}7 \\
(4,1 \%)\end{array}$ & 0 & 0 & 0 & $\begin{array}{c}7 \\
(1,9 \%)\end{array}$ \\
\hline & $\mathrm{p}$ & & \multicolumn{5}{|c|}{0,001} \\
\hline
\end{tabular}

TENS: técnicas/os de enfermería de nivel superior.

* Mediana de la edad en funcionarios/as que muestran diferencias significativas.

Fuente: elaboración propia a partir de información obtenida por Ley 20.285 .

Tabla 5. Accesibilidad a la interrupción voluntaria del embarazo en tres causales según porcentaje de objetores/as en hospitales públicos. Chile, junio de 2020

\begin{tabular}{|c|c|c|c|c|c|c|c|c|c|c|c|c|}
\hline \multicolumn{13}{|c|}{ Número de establecimientos } \\
\hline Profesión & \multicolumn{3}{|c|}{ Gineco-obstetras } & \multicolumn{3}{|c|}{ Anestesistas } & \multicolumn{3}{|c|}{$\begin{array}{l}\text { Profesionales no } \\
\text { médicos }\end{array}$} & \multicolumn{3}{|c|}{ TENS/auxiliares } \\
\hline Causales & 1 & 2 & 3 & 1 & 2 & 3 & 1 & 2 & 3 & 1 & 2 & 3 \\
\hline $0-25 \%$ & 35 & 31 & 12 & 42 & 40 & 31 & 47 & 44 & 37 & 46 & 45 & 40 \\
\hline $25,1-50 \%$ & 17 & 18 & 14 & 10 & 12 & 19 & 4 & 6 & 9 & 5 & 5 & 8 \\
\hline $50,1-75 \%$ & 4 & 5 & 15 & 1 & 1 & 2 & 1 & 2 & 4 & 2 & 3 & 3 \\
\hline $75,1-100 \%$ & 1 & 3 & 16 & 1 & 1 & 2 & 2 & 2 & 3 & 1 & 2 & 3 \\
\hline Total hospitales & 57 & 57 & 57 & 54 & 54 & 54 & 54 & 54 & 53 & 54 & 55 & 54 \\
\hline
\end{tabular}

TENS: técnicas/os de enfermería de nivel superior.

Fuente: elaboración propia a partir de información obtenida por Ley 20.285.

la reprobación social y profesional, derivado del estigma hacia el aborto ${ }^{18}$.

Es factible reconocer argumentos en cada causal. Frente al riesgo vital materno y la inviabilidad embrionaria/fetal letal, emerge el embrión/feto como paciente y su necesaria protección. En esta última, el embrión/feto sería un enfermo terminal, que amerita cuidados paliativos ${ }^{18}$, enarbolándose incluso razones 


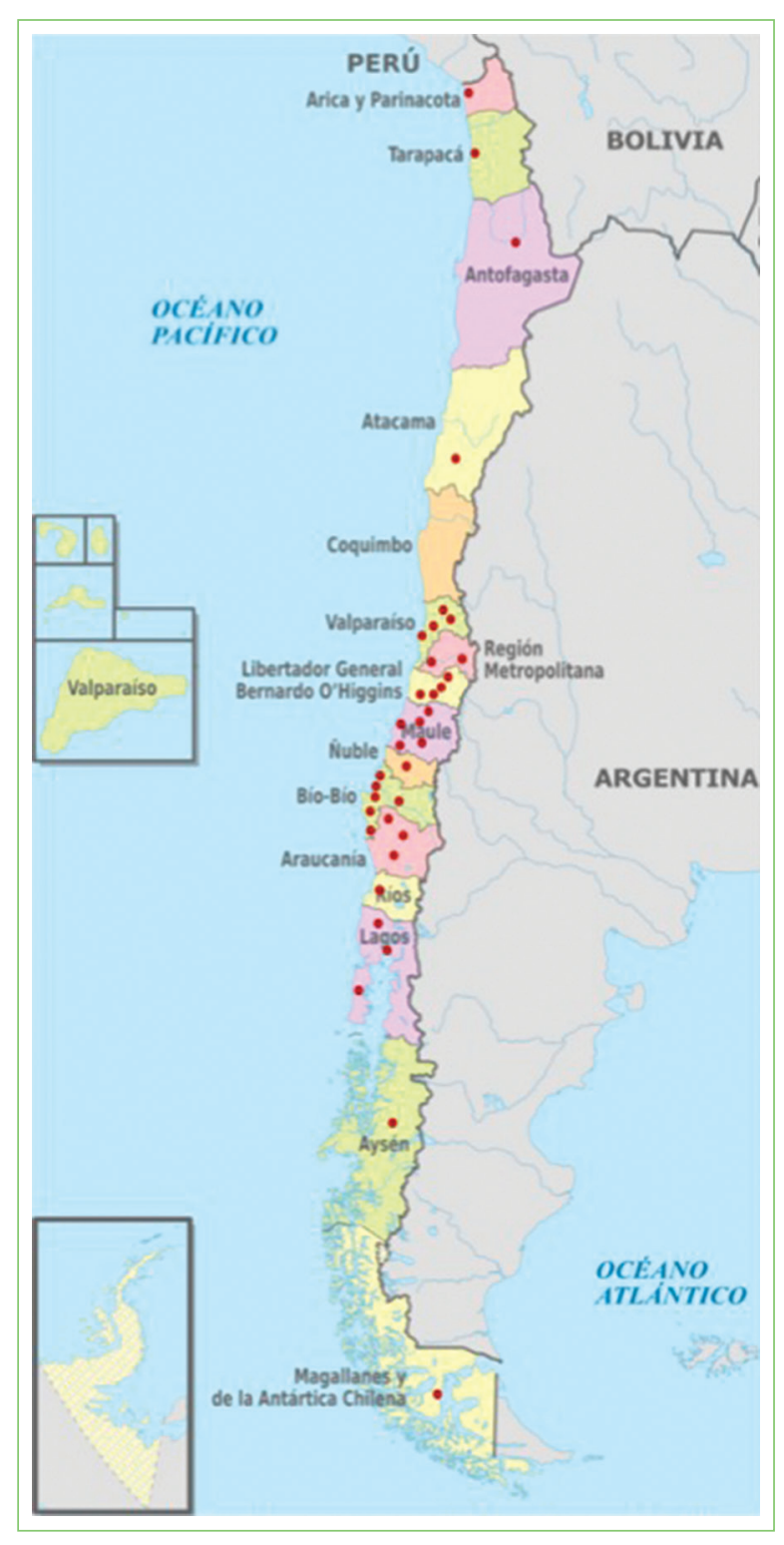

Figura 1. Georreferenciación de hospitales públicos con proporción de objetores/as de conciencia superior al $50 \%$. Chile, junio de 2020 . Fuente: elaboración propia a partir de información obtenida por Ley 20.285.

técnicas vinculadas con las dificultades para obtener un diagnóstico certero. En el embarazo por violación, existe un discurso paternalista y estigmatizante que trivializa la violación y cuestiona el relato de la mujer; se considera a la gestante y al embrión/feto sin patologías biomédicas que justifiquen la interrupción y se valora al embrión/feto como víctima, cuyo derecho a la vida prevalecería sobre el derecho de la mujer a la IVE $^{18,20}$.

En Uruguay se identificaron objetores donde primaba su conciencia por sobre el deber profesional, sin obstaculizar el ejercicio del derecho de las mujeres a la IVE, y objetores que según sus creencias, principalmente religiosas, obstaculizaban el ejercicio de este derecho. Los pseudoobjetores se caracterizaban por utilizar la objeción por razones de conveniencia personal, sin un fundamento claro ni acorde al dictamen de su conciencia moral ${ }^{11}$. Al igual que en Uruguay, en Chile existirían otros fundamentos ajenos a la conciencia moral, dando origen a pseudoobjeciones de conciencia, lo que es necesario objetivar investigando con mayor profundidad en este ámbito. Küng et al. ${ }^{10}$ señalan como razones no morales para objetar la falta de conocimiento de la normativa legal y el temor para enfrentar eventuales problemas legales derivados de la provisión de servicios de aborto.

\section{Conclusiones}

Las dificultades para obtener información sobre objetores desde las instituciones de salud pertenecientes a la Administración del Estado no permiten conocer a cabalidad lo que está sucediendo en Chile al interior de los equipos, dando cuenta de una importante barrera en la implementación de la Ley 21.030. Aun cuando la objeción de conciencia es reconocida en esta ley, no puede ser utilizada para eludir responsabilidades profesionales ni puede operar como un obstáculo que vulnere los derechos fundamentales y la dignidad de las mujeres que resulten afectadas por su invocación.

Las directrices éticas de la Federación Internacional de Ginecología y Obstetricia promueven el compromiso de conciencia, estableciendo como deber primario la obligación del profesional de promover el bienestar, cautelar el resguardo de la salud e impedir la ocurrencia de posibles daños de las pacientes, resultando la objeción secundaria a este deber primordial ${ }^{21,22}$. Es perentorio establecer perfiles de cargo en la contratación de funcionarios/as para garantizar las prestaciones legalmente establecidas. Junto con capacitar sobre el marco legal, debieran fomentarse intervenciones al interior de los equipos y en la formación de las nuevas generaciones de profesionales y técnicos en salud, que posibiliten realizar un examen de conciencia, con miras a reflexionar sobre los motivos para objetar, sobre su vocación de servicio público y deber profesional, analizando las implicancias que la objeción determine para la salud y los derechos de las mujeres, especialmente de aquellas que no tienen la posibilidad de elegir el establecimiento ni el/la profesional para su atención. 


\section{Agradecimientos}

A las personas funcionarias de las instituciones de salud que compilaron y compartieron la información. Al proyecto de investigación del cual deriva este manuscrito: Fondecyt Regular oㅜ 1200374 "Representaciones sociales de actores relevantes vinculadas con las principales barreras a las prestaciones garantizadas en la Ley 21.030, que despenaliza la interrupción del embarazo por tres causales en Chile".

\section{Financiamiento}

Fondo Nacional de Desarrollo Científico y Tecnológico (FONDECYT), de la Agencia Nacional de Investigación y Desarrollo (ANID).

\section{Conflicto de intereses}

Las autoras y el autor declaran no tener ningún conflicto de intereses para efectos de esta publicación.

\section{Responsabilidades éticas}

Protección de personas y animales. Los autores declaran que para esta investigación no se han realizado experimentos en seres humanos ni en animales.

Confidencialidad de los datos. Los autores declaran que en este artículo no aparecen datos de pacientes.

Derecho a la privacidad y consentimiento informado. Los autores declaran que en este artículo no aparecen datos de pacientes.

\section{Bibliografía}

1. Ley $n .{ }^{\circ}$ 21.030: Regula la despenalización de la interrupción voluntaria del embarazo en tres causales. Disponible en: http://www.diariooficial. interior.gob.cl/publicaciones/2017/09/23/41866/01/1276248.pdf

2. Montero A, Vergara J, Ríos M, Villarroel R. La objeción de conciencia en el debate sobre la despenalización del aborto por tres causales en Chile. Rev Chil Obstet Ginecol. 2017;82:350-60.

3. Tribunal Constitucional de Chile. Sentencia del Tribunal Constitucional sobre el proyecto de ley que regula la despenalización de la interrupción voluntaria del embarazo en tres causales. Boletín N. ${ }^{\circ}$ 9895-11. ROL 3739-17". (Consultado el 08/01/2019.) Disponible en: https://www.tribunalconstitucional.cl
4. Ministerio de Salud, Gobierno de Chile. Protocolo para la manifestación de objeción de conciencia personal y para la objeción de conciencia invocada por instituciones en el marco de lo dispuesto en el artículo 119 ter del Código Sanitario. Aprobado mediante la Resolución Exenta n. ${ }^{\circ} 61$ del 22 de enero de 2018, publicada en el Diario Oficial el día 27 de enero de 2018. (Derogado). (Consultado el 30/04/2021.) Disponible en: http://www.minsal. cl/wp- content/uploads/2018/02/Protocolo Objecion Conciencia.pdf

5. Ministerio de Salud, Gobierno de Chile. Protocolo para la manifestación de la objeción de conciencia según lo dispuesto en el artículo 119 ter del Código Sanitario. Aprobado mediante Resolución Exenta n. ${ }^{\circ} 432$ del 22 de marzo de 2018. (Derogado). (Consultado el 30/04/2021.) Disponible en: https://www.minsal.cl/wp-content/uploads/2018/02/Protocolo.pdf

6. Robledo P. Objeción de conciencia: entre libertades y derechos. Rev Chil Salud Pública. 2018:22:179-87.

7. Reglamento para ejercer objeción de conciencia según lo dispuesto en el artículo 119 ter del Código Sanitario. (Consultado el 30/04/2021.) Disponible en: http://www.diariooficial.interior.gob.cl/publicaciones/2018/10/23/42187/01/1482452.pdf

8. Casas L, Dides C. Objeción de conciencia y salud reproductiva en Chile: dos casos paradigmáticos. Acta Bioethica. 2007;13:199-206.

9. Giubilini A, Savulescu J. Beyond money: conscientious objection in medicine as a conflict of interests. Bioethical Inquiry. 2020;17:229-43.

10. Küng S, Danette J, Díaz de León F, Huaraz F, Pearson E. We don't want problems: reasons for denial of legal abortion based on conscientious objection in Mexico and Bolivia. Reprod Health. 2021;18:44. Disponible en: https://doi.org/10.1186/s12978-021-01101-2.

11. Coppola F, Briozzo L, Nozar F, Fiol V, Greif D. Conscientious objection as a barrier for implementing voluntary termination of pregnancy in Uruguay: gynecologists' attitudes and behavior. Int J Gynecol Obstet. 2016;134:S16-9.

12. Ministerio de Salud, Gobierno de Chile. Funcionarios objetores por Servicio de Salud, 2019. (Consultado el 18/08/2021.) Disponible en: https:// www.minsal.cl/funcionarios-objetores-de-conciencia-por-servicio-de-salud

13. Ministerio de Salud, Gobierno de Chile. Funcionarios objetores por Servicio de Salud, 2018. (Consultado el 28/11/2018.) Disponible en: https:// www.minsal.cl/funcionarios-objetores-de-conciencia-por-servicio-de-salud

14. Ministerio de Salud, Gobierno de Chile. Listado de objetores institucionales. (Consultado el 30/04/2021.) Disponible en: https://www.minsal.cl/ listado-de-objetores-institucionales

15. Ley $n .^{\circ}$ 20.285: Regula el acceso a la información pública. (Consultado el 29/04/2021.) Disponible en: https://www.bcn.cl/leychile/navegar?idNor$\mathrm{ma}=276363$

16. Maturana C. Corporación Humanas: Implementación de la Ley No 21.030 que regula la despenalización de la interrupción voluntaria del embarazo en tres causales: Objeción de conciencia en hospitales públicos y en instituciones y su impacto en el ejercicio de derechos de las mujeres y niñas. (Consultado el 25/07/2021.) Disponible en: http://www.humanas. cl/wp-content/uploads/2018/08/HUMANAS-Estudio-Objeción-Conciencia-Ley-IVE-Tres-Causales-Anexo-Julio-2018.pdf

17. Ministerio de Salud, Gobierno de Chile. Reporte Ley 21.030: Número de casos por causal constituida. (Consultado el 30/04/2021.) Disponible en: https://informesdeis.minsal.cl/SASVisualAnalytics/?reportUri=\%2Freports\%2Freports\%2F382105c8-521f-4356-b1b8-6bad21ba8b08\&sectionIndex=0\&sso_guest=true\&reportViewOnly=true\&reportContextBar=false\&sas-welcome=false

18. Montero A, Ramírez-Pereira M. Noción y argumentos sobre la objeción de conciencia al aborto en Chile. Revista Bioética y Derecho. 2020;49:59-75.

19. Casas L, Freedman L, Ramm A, Correa S, Baba F. Chilean medical and midwifery faculty's views on conscientious objection for abortion services. Int Perspect Sex Reprod Health. 2020;46 (Suppl 1):25-34.

20. Ramírez-Pereira M, Montero A. Representaciones sociales sobre género en actores relevantes vinculadas con la despenalización de la interrupción del embarazo en Chile. Atenea. 2021;523:331-45.

21. Faúndes A, Alves G, Duarte M. Conscientious objection or fear of social stigma and unawareness of ethical obligations. Int J Gynecol Obstet. 2013;123:S57-9.

22. FIGO Committee for the Study of Ethical Aspects of Human Reproduction and Women's Health. Ethical guidelines on conscientious objection. En: Ethical issues in obstetrics and gynecology. October 2012. (Consultado el 30/04/2021.) Disponible en: https://www.glowm.com/pdf/English\%20 Ethical\%20lssues\%20in\%20Obstetrics\%20and\%20Gynecology.pdf 